\title{
Field-emission scanning probe lithography with self-actuating and self-sensing cantilevers for devices with single digit nanometer dimensions
}

Ivo W. Rangelow, Claudia Lenk, Martin Hofmann, Steve Lenk, Tzvetan Ivanov, et al.

Ivo W. Rangelow, Claudia Lenk, Martin Hofmann, Steve Lenk, Tzvetan Ivanov , Ahmad Ahmad, Marcus Kaestner, Elshad Guliyev, Christoph Reuter, Matthias Budden, Jens-Peter Zöllner, Mathias Holz, Alexander Reum, Zahid Durrani, Mervyn Jones, Cemal Aydogan, Mahmut Bicer, B. Erdem Alaca, Michael Kuehnel, Thomas Fröhlich, Roland Fuessl, E. Manske, "Fieldemission scanning probe lithography with self-actuating and self-sensing cantilevers for devices with single digit nanometer dimensions," Proc. SPIE 10584, Novel Patterning Technologies 2018, 1058406 (19 March 2018); doi: $10.1117 / 12.2299955$ 


\title{
Field-Emission Scanning Probe Lithography with self-actuating and self-sensing cantilevers for devices with single digit nanometer dimensions
}

Ivo W. Rangelow*a, Claudia Lenk ${ }^{\text {a }}$, Martin Hofmann ${ }^{\text {a, Steve Lenk }}{ }^{\text {a }}$, Tzvetan Ivanov ${ }^{\text {a }}$, Ahmad Ahmad

a, Marcus Kaestner ${ }^{\text {a }}$, Elshad Guliyev a , Christoph Reuter ${ }^{\text {a }}$, Matthias Budden ${ }^{\text {a }}$, Jens-Peter Zöllner ${ }^{a}$

a Ilmenau University of Technology, Institute of Micro- and Nanoelectronics, Department of Micro- and Nanoelectronic Systems (MNES), Gustav-Kirchhoff-Str.1, 98693 Ilmenau, Germany

Mathias Holz ${ }^{\mathrm{b}}$, Alexander Reum ${ }^{\mathrm{b}}$

bNano Analytik GmbH, Ehrenbergstraße 1, 98693 Ilmenau, Germany

Zahid Durrani $^{\mathrm{c}}$, Mervyn Jones ${ }^{\mathrm{c}}$

${ }^{c}$ Department of Electrical and Electronic Engineering, Imperial College London, South Kensington, London SWT 2AZ, United Kingdom

Cemal Aydogan ${ }^{\mathrm{d}}$
${ }^{\mathrm{d}}$ Electronics and Cryptology Department TUBITAK - YITAL, Gebze, Kocaeli, Turkey

Mahmut Bicer ${ }^{\mathrm{e}}$, and B. Erdem Alaca ${ }^{\mathrm{e}}$

e Department of Mechanical Engineering, Koc University, Rumelifeneri Yolu, Sariyer, 34450 Istanbul, Turkey

Michael Kuehnel ${ }^{f}$, Thomas Fröhlich ${ }^{\mathrm{f}}$, Roland Fuessl ${ }^{\mathrm{f}}$, and E. Manske ${ }^{\mathrm{f}}$

f Institute of Process Measurement and Sensor Technology, TU Ilmenau, Gustav-Kirchhoff-Str.

1, 98693 Ilmenau, Germany

*E-mail: ivo.rangelow@tu-ilmenau.de

\begin{abstract}
Cost-effective generation of single-digit nano-lithographic features could be the way by which novel nanoelectronic devices, as single electron transistors combined with sophisticated CMOS integrated circuits, can be obtained. The capabilities of Field-Emission Scanning Probe Lithography (FE-SPL) and reactive ion etching (RIE) at cryogenic temperature open up a route to overcome the fundamental size limitations in nanofabrication. FE-SPL employs Fowler-Nordheim electron emission from the tip of a scanning probe in ambient conditions. The energy of the emitted electrons $(<100 \mathrm{eV})$ is close to the lithographically relevant chemical excitations of the resist, thus strongly reducing proximity effects. The use of active, i.e. self-sensing and self-actuated, cantilevers as probes for FE-SPL leads to several promising performance benefits. These
\end{abstract}


include: (1) Closed-loop lithography including pre-imaging, overlay alignment, exposure, and post-imaging for feature inspection; (2) Sub-5-nm lithographic resolution with sub-nm line edge roughness; (3) High overlay alignment accuracy; (4) Relatively low costs of ownership, since no vacuum is needed, and ease-of-use. Thus, FE-SPL is a promising tool for rapid nanoscale prototyping and fabrication of high resolution nanoimprint lithography templates. To demonstrate its capabilities we applied FE-SPL and RIE to fabricate single electron transistors (SET) targeted to operate at room temperature. Electrical characterization of these SET confirmed that the smallest functional structures had a diameter of only 1.8 nanometers. Devices at single digit nano-dimensions contain only a few dopant atoms and thus, these might be used to store and process quantum information by employing the states of individual atoms.

Keywords: Scanning Probe Lithography, SPL, nanofabrication, calixarene, molecular glass resist, closed loop lithography, nanoprobe maskless lithography, self-developing resist

\section{INTRODUCTION}

Fabrication of devices with single digit nanometer dimensions requires high resolution, reproducible lithography, high precision positioning of patterns with respect to predefined structures, high fidelity pattern transfer to preserve the nano-sized features and metrology for the nanometer scale. These issues can be addressed by using Field-Emission Scanning Probe Lithography (FE-SPL) with self-actuating and self-sensing cantilevers combined with cryogenic reactive ion etching (cRIE) ${ }^{1}$. FE-SPL, on the one hand, provides pattern generation with nanometer precision, pattern overlay alignment, critical dimension (CD) measurement and inspection (imaging). On the other hand, cRIE enables highly anisotropic transfer of single-digit nanometer sized patterns written in thin resist layers $(\sim 10 \mathrm{~nm})$ into silicon. With respect to achieved resolution, throughput, overlay alignment accuracy, defect density, cost of ownership, and reliability FE-SPL method is considered as a promising candidate for a widespread use in device prototyping or template fabrication for nanoimprint lithography (NIL). Several hybrid lithographic tip-based patterning methods have been developed using: (i) force induced interactions such as plowing $^{2}$, nanografting ${ }^{3}$, and atomic manipulation ${ }^{4}$; (ii) heat-induced thermomechanical ${ }^{5}$ or thermochemical ${ }^{6}$ interactions; $^{2}$; (iii) electric-field-induced interactions such as electrochemical modification or deposition ${ }^{7}$, and resist exposure applying lowenergy electrons ${ }^{8}$. Scanning Tunneling Microscope based surface patterning experiments have shown the potential of atom manipulation $^{9}$ and the utility of the probe as a local miniature emission source for exposure of various resist materials ${ }^{10}$. During the last decade, remarkable developments in lithography with unique resolution capabilities have been demonstrated ${ }^{11}$. These tip-based technologies can enable a range of innovative nanoscale devices to be made and have introduced a number of new nanotechnology-based products to the market. For these devices to become the basis for high value nanoelectronic technology, lithographic methods must be established which can efficiently produce systems that integrate together billions of nanoscale devices with dissimilar functions ${ }^{12}$. Currently sub-10nm lithography such as those demonstrated in the semiconductor research laboratories will not be economical at these scales and throughput; radically new mass production techniques are needed. To address this need, we use a mix\&match technology for fabrication of highperformance nanoimprint lithography templates for high-throughput production ${ }^{13,14}$. Thereby, all larger non-CD features are defined by electron beam lithography (EBL) and, in a subsequent lithography step, all CD features (below $20 \mathrm{~nm}$ ) are written by closed loop FE-SPL.

In this paper we present results from the fabrication of nanoscale features and devices by FE-SPL and high-resolution pattern transfer using reactive plasma etching at cryogenic temperatures. As a demonstrator of the success of this process chain, we have fabricated single electron transistors, which were shown to operate at room temperature.

\section{EXPERIMENTAL DETAILS}

The scanning probe lithography experiments were carried out with a dedicated FE-SPL tool, shown in Fig. 1, which is operated in well controlled ambient conditions with relative humidity $(30 \% \pm 10 \%)$ and room temperature $\left(21^{\circ} \pm 2^{\circ} \mathrm{C}\right)$. For 
lithography, electrons are emitted from a sharp tip of a scanning probe (Fig. 1b) due to a bias voltage between tip and sample $(<100 \mathrm{~V})$. The emission process thereby follows the Fowler-Nordheim dependency. Thus, the emission current can be regulated by changing the distance between tip and sample or the bias voltage. Since the tip-sample distance is in the range of $10-100 \mathrm{~nm}$ and the mean free path of the electrons in air is $>100 \mathrm{~nm}$, the system can be operated at ambient conditions without the requirement for a vacuum environment. The emitted electrons are used to expose ultrathin resist layers with typical thicknesses in the range of 5-20 nm. The energy of the electrons is in the range of several $10 \mathrm{eV}$, which is comparable

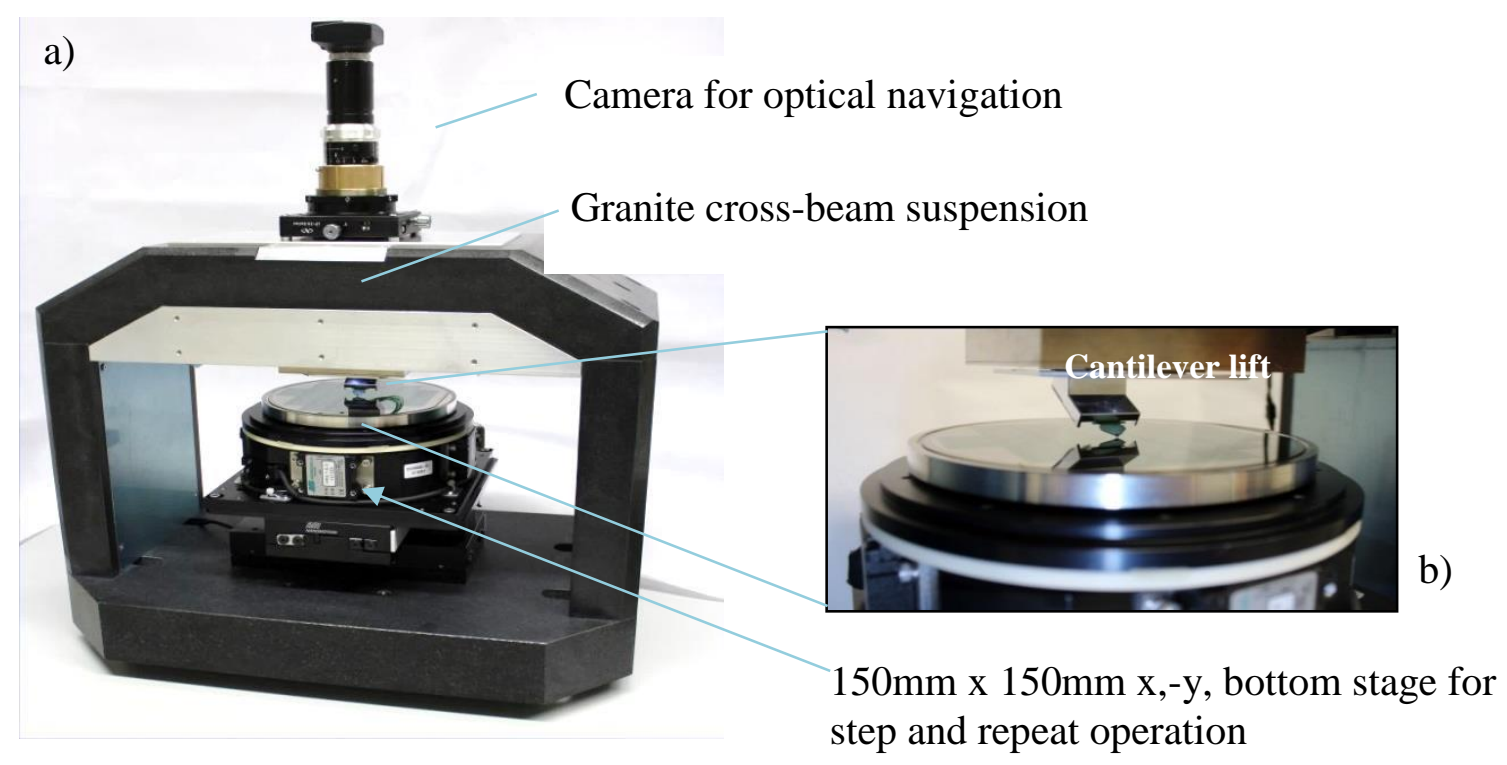

c)

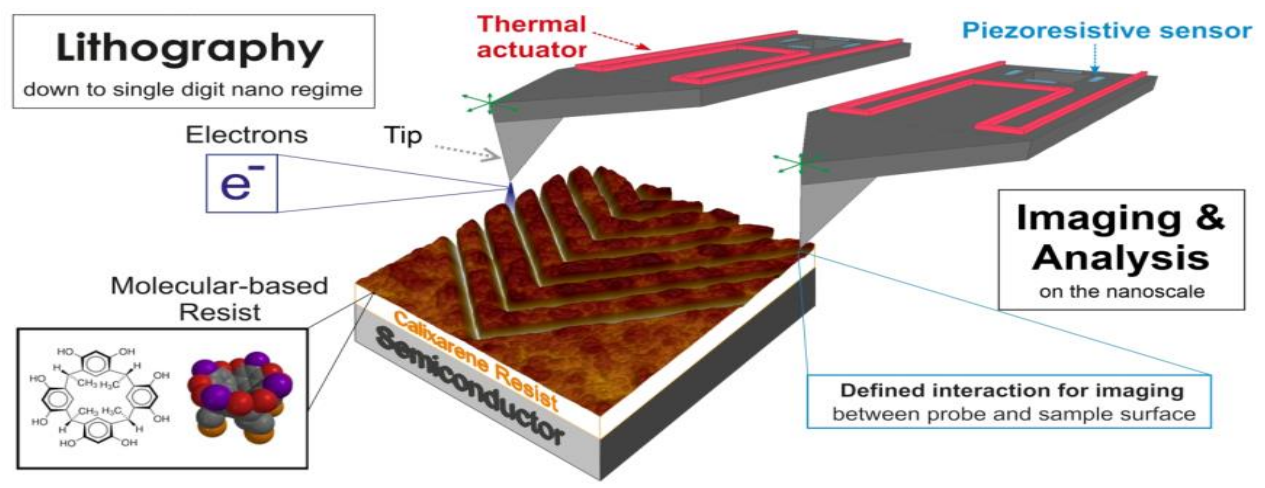

Figure 1. Set-up for Field-Emission Scanning Probe Lithography (FE-SPL). (a)-(b) FE-SPL tool developed in the Rangelow group, including camera for optical navigation, $(150 \mathrm{~mm} \times 150 \mathrm{~mm})$ bottom stage for step and repeat operation and a granite cross-beam suspension holding the ( $x=200 \mu \mathrm{m} ; \mathrm{y}=200 \mu \mathrm{m} ; \mathrm{z}=20 \mu \mathrm{m}$ top-scanner with the active cantilever (green) and electronics. (c) Principle setup of SPL tool with current feedback loop for FE-SPL and a force feedback loop for AFM imaging. Switching between modes (imaging and lithography) is possible, thereby using the same nanoprobe for both, direct writing of nanofeatures using a low-energy FN-field-electron emission from nano-probe-tip and AFM-imaging for pre- and post-inspection, as well as for pattern overlay alignment [from ${ }^{15}$ ]

to the relevant chemical excitations of the resist. Proximity effects, as observed for EBL due to the generation of secondary electrons, are strongly reduced for FE-SPL. Positive (bond breaking) as well as negative tone (cross-linking) can be obtained 
after development of the exposed structures, as shown in Fig. 2 (a)-(c). Furthermore, FE-SPL provides a self-development positive tone mode, for which resist material is removed without the requirement of a development step (see Fig. 2c). The exposure dose, i.e. the lithography current divided by the writing speed, acts as a switch between the different lithographic tones. The most frequently selected resist for FE-SPL is calixarene ${ }^{1}$, offering high-resolution patterning due to its small molecule size and low surface roughness. Furthermore, for non-conducting samples, the conducting resist P3HT can be used, which, in this case, enables a conducting pathway for the emission current. Thus, there are no restrictions for the applicability of FE-SPL regarding the conductivity of the samples. Resistless lithography is enabled if FE-SPL is used to oxidize the sample (e.g. Cr, W).

For high resolution lithography and imaging, tip shape and tip radius become crucial. In both cases, resolution is determined primarily by the sharpness of the tip. The tip-radius of curvature is approximately $5 \mathrm{~nm}$ (see Fig. $3 \mathrm{~b}$ ), enhancing the resolution capability for imaging and lithography significantly. Furthermore, resolution depends on the exposure dose, i.e. increasing the exposure dose results in larger features.

a)
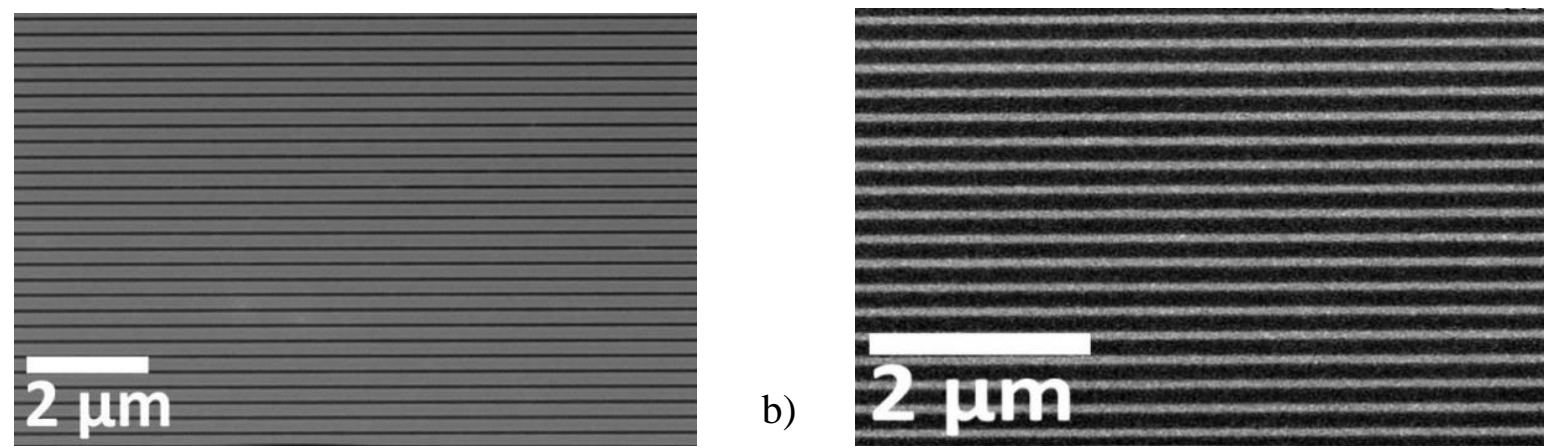

\section{b)}

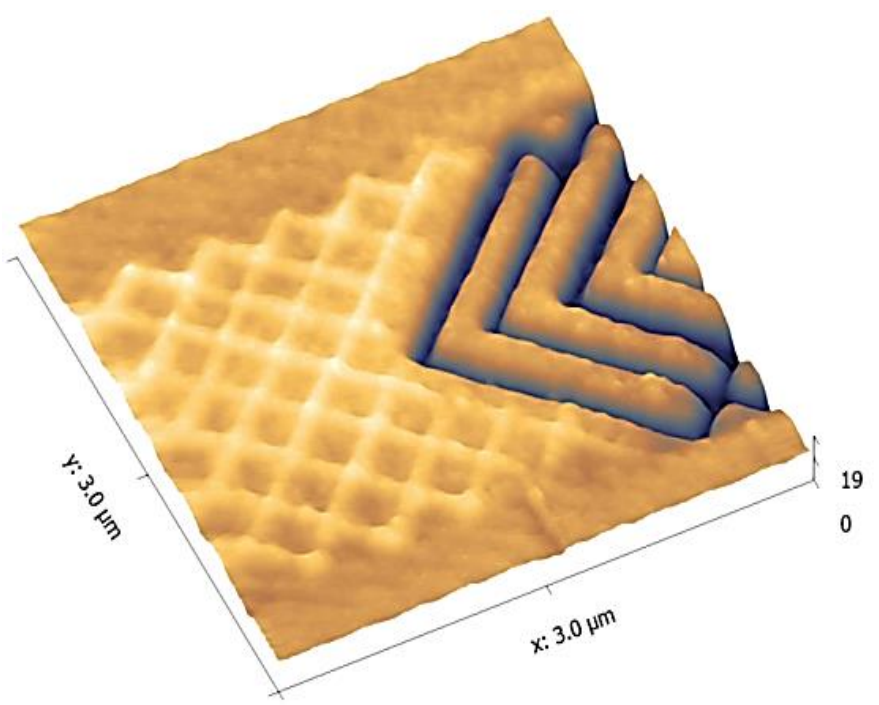

Figure 2. Demonstration of switching of lithographic tones by FE-SPL. (a)-(b) SEM images of (a) negative and (b) positive tone of lines after development. All features were written into $5 \mathrm{~nm}$ thick cmc4r resist layer (line pitch of 250nm). The exposure doses were chosen with respect to the targeted lithographic tones $(25 \mathrm{nC} / \mathrm{cm}$ for the negative tone and $142 \mathrm{nC} / \mathrm{cm}$ for the positive tone). (c) AFM image showing a single corner feature array patterned in negative and positive tone (without development). The exposure dose for negative tone was 44 $\mathrm{nC} / \mathrm{cm}$. The same lines ware overwritten (secondary exposure of the same lines) with $120 \mathrm{nC} / \mathrm{cm}$ resulting in positive tone. 
a)

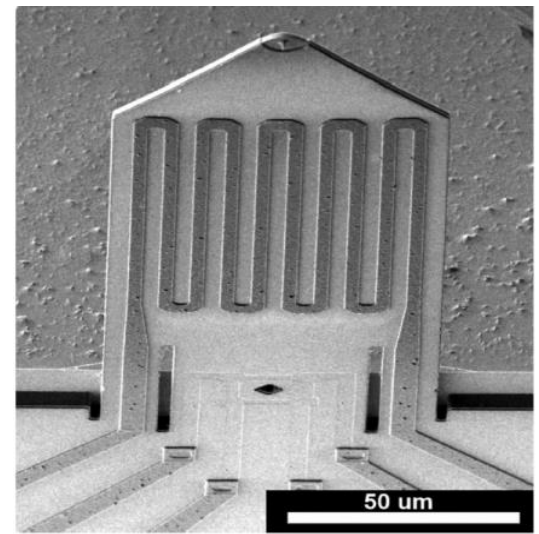

b)

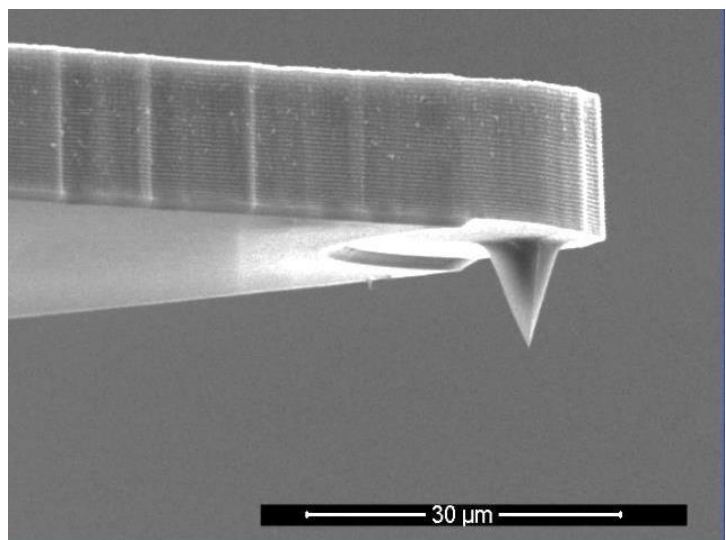

Fig. 3: (a) SEM images of active cantilever showing aluminium meander for thermomechanical actuation and piezoresistors for deflection measurement [from ${ }^{1}$ ]. (b) SEM image of sharp silicon tip at the front of the active cantilever. Typical tip radius is 5-10 $\mathrm{nm}$.

An important advantage in the performance of the FE-SPL technology arises from the fact that the same active-cantilever [Fig. 3(a)+(b)] is used for both, direct low-energy Fowler-Nordheim electron exposure of the resist using field emission from the tip and fast noncontact atomic force microscopy (AFM). The active cantilevers are based on well-established thermomechanical actuation principles and a self-sensing scheme, demonstrated and described previously ${ }^{16,17}$. Cantilever deflection and oscillation amplitude can be precisely controlled by dissipated electrical power within resistors on top of the cantilever (see Fig. 3(a)). In this way, resonance and static cantilever deflection can be excited ${ }^{18,19}$. The deflection amplitude of the cantilever vibration as well as the static bending of the cantilever is determined by acquisition of the output signal of integrated piezoresistive deflection sensors ${ }^{18}$. These form an integrated Wheatstone-bridge at the point of highest internal mechanical stress, enabling deflection detection at low thermal noise.

To enable both modes, FE-SPL and AFM to be used, two control feedback loops are implemented in the FE-SPL tool and presented schematically in Fig. 1(c). The closed-loop FE-SPL tool uses the standard dynamic amplitude modulation feedback loop for topographic imaging (AFM). Thereby, the height of the cantilever is regulated by a scanner on the basis of the difference of the oscillation amplitude to a predefined amplitude set-point. The imaging is performed routinely with atomic resolution and employed for pre- and post-inspection. This enables precise pattern overlay alignment and feature stitching ${ }^{20}$, as shown for a representative example in Fig. 4. For FE-SPL mode, a second independently working feedback loop is used, which regulates the height of the cantilever to maintain the current set-point. To control the electron emission current required for the lithography feedback loop, a two-stage high precision current-to-voltage (IV)-converter with subsequent amplification stage was developed. The low-noise preamplifier is characterized by a transfer function of $5 \mathrm{~V} / \mathrm{nA}$ and $0.07 \mathrm{pA}$ noise level at $1.5 \mathrm{kHz}$ bandwidth.

The active cantilever approach facilitates the implementation of a compact, table-top scanning probe lithography tool (see Fig. 1). Scanner with $10 \times 10 \mu \mathrm{m}^{2}$ or $200 \times 200 \mu \mathrm{m}^{2}$ scan area can be used. To reduce thermal drift and enable a high mechanical stability, the top scanner is mounted on a cross-beam suspension made from granite. While AFM imaging is used for precise positioning and alignment, a camera mounted on top of the system is used for coarse positioning by optical navigation. To enable an increased patterning area of up to $100 \times 100 \mathrm{~mm}^{2}$, a step-and-repeat function is implemented, with 
which the same structure can be written repeatedly on the sample by movement of the bottom stage between subsequent lithography steps.

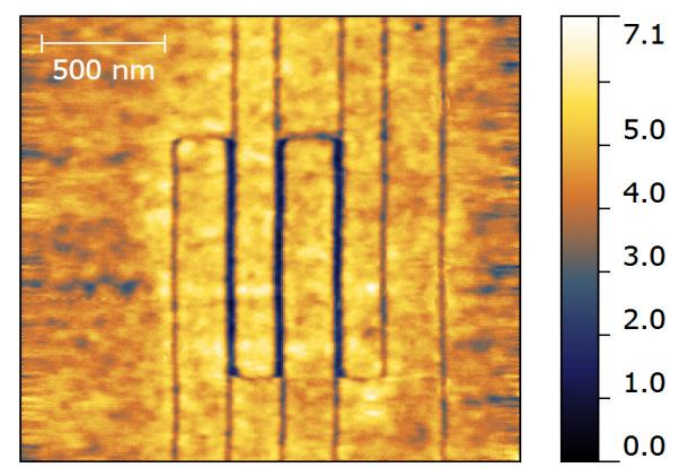

Figure 4: Demonstration of overly accuracy. AFM image shows meander-pattern obtained in two exposure steps. First-meander was exposed with $82 \mathrm{nC} / \mathrm{cm}$ and second meander with the same dose and positioned exactly over the first one using AFM-alignment mode. This results in positive tones (three dark lines in the middle of the image).

The combination of high resolution non-contact AFM imaging (used also for pattern inspection and placement) with high resolution low energy electron exposure makes the FE-SPL-tool irreplaceable for many nano-lithographic applications. A precise overlay alignment without the requirement of marks is possible. The minimization of thermal drift effects is achieved by keeping the sample in the tool for sufficient time before processing to reach thermal equilibrium and by reducing the time between AFM navigation and exposure using a fast switching principle. The mechanical drift of the piezo-stack stage is compensated due to measuring the placement of the features shortly before exposure. The simplicity, controllability, and reproducibility of the combination of these methods (FE-SPL, AFM) are enabling a new perspective to be opened for the easy fabrication of beyond CMOS devices, with the added attraction of not requiring a high capital investment.

\section{PATTERN TRANSFER BY CRYOGENIC ETCHING}

In terms of FE-SPL we require resist layers in the range of $10 \mathrm{~nm}$ in order to be able to generate single nano digit features. Herein, the challenge is associated with the transfer of features into silicon giving practical selectivities, process control and etching depths for device manufacturing. For pattern transfer in the nanoscale range, a cryogenic etching procedure is used. This process, which had been introduced by Tachi et al. ${ }^{21}$, then followed by many studies ${ }^{22-24}$, revealed enhancements compared to the standard reactive ion etching at room temperature.

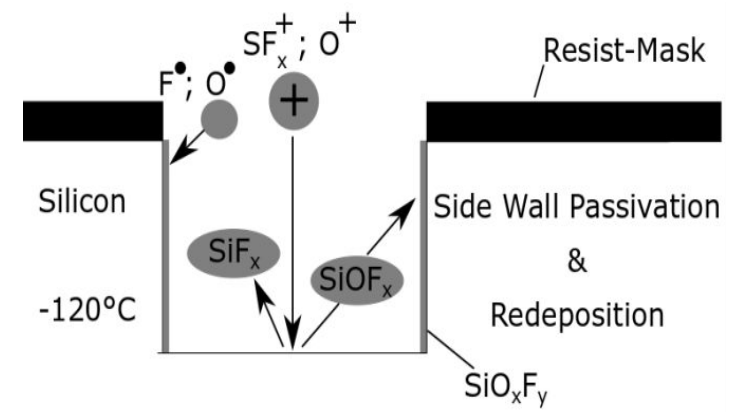

Figure 5. Demonstration of the basic principle of ions and radicals involved in cryoetching of $\mathrm{Si}$ using $\mathrm{SF}_{6} / \mathrm{O}_{2}$ gases with the accompanying side wall passivation [from ${ }^{25}$ ]. 
Figure 5 depicts the principle of cryogenic etching. Basically, the idea of cryogenic etching is to use the effect of ionized feed gases at low substrate temperatures ${ }^{26}$. Here, $\mathrm{SF}_{6} / \mathrm{O}_{2}$ gases at cryogenic temperatures are used, which are typically applied for deep anisotropic etching of silicon. Due to the exposure to fluorine, silicon is chemically etched and volatile products are generated. In 1991, Winters and Plumb ${ }^{27}$ found the primary products $\mathrm{SiF}_{4}, \mathrm{Si}_{2} \mathrm{~F}_{6}$ and $\mathrm{Si}_{3} \mathrm{~F}_{8}$, with $\mathrm{SiF}_{4}$ as the main product. In their studies, they found no evidence of $\mathrm{SiF}_{2}$ being a prior product at room temperature. However, other groups, have concluded that while $\mathrm{SiF}_{4}$ was the main product, they also found a significant amount of $\mathrm{SiF}_{2}$ in the gas phase ${ }^{28,29}$. This disagreement is still not resolved; hence further studies are needed to answer this question ${ }^{30}$. Whether or not $\mathrm{SiF}_{2}$ is an etching product, the resulting etching behavior of silicon by pure fluorine feed gases is isotropic, thus not feasible for pattern transfer at the nano scale.

By the addition of oxygen to the gas mixture, recombination of $\mathrm{Si}, \mathrm{O}$, and $\mathrm{F}$ becomes energetically favorable and $\mathrm{SiO}_{\mathrm{x}} \mathrm{F}_{\mathrm{y}}$ molecules are created. This polymer is volatile at room temperature. By lowering the temperature below $-100{ }^{\circ} \mathrm{C}$, the sticking probabilities of the reactants increase and enable the formation of a polymeric layer of $\mathrm{SiO}_{\mathrm{x}} \mathrm{F}_{\mathrm{y}}$ on the silicon surface ${ }^{31}$. This protection layer hinders the fluorine from attacking the $\mathrm{Si}$. Due to the condensation of $\mathrm{Si}_{2} \mathrm{~F}_{6}$ on the silicon surface, the spontaneous etching of Si by fluorine is blocked at temperatures lower than $-196^{\circ} \mathrm{C}^{32}$, setting a lower limit for the practically usable temperatures. The typical substrate temperature range used for cryogenic etching is between $-100{ }^{\circ} \mathrm{C}$ to $-140{ }^{\circ} \mathrm{C}^{26,31}$. At this point etching would be expected to stop as a result of the passivation layer $\left(\mathrm{SiO}_{\mathrm{x}} \mathrm{F}_{\mathrm{y}}\right)$, since it prevents further etching in both vertical and horizontal direction. To circumvent this, sputtering is applied in addition to the chemical etching. Therefore, a voltage difference between plasma bulk and sample surface is used, which triggers ions from the plasma to be accelerated straight towards the sample. Depending on the actual process conditions, the energy of these ions is in the range of a few tens of electron-volts ${ }^{33}$, which is enough energy to remove the passivation layer on the horizontal silicon surface by the impinging ions. Due to the glancing angle of incidence on the feature sidewalls, there is low or no sputtering of the vertically deposited passivation layer. Additionally, the physical sputtering due to the ion bombardment also prevents the growth of an inhibitor film. Thus, fluorine can react spontaneously with silicon on the bottom of the feature, forming volatile $\mathrm{SiF}_{4}$ molecules which desorb from the surface. As a result of these processes, the etching proceeds only in vertical direction (ion bombardment direction) and an anisotropic profile can be achieved ${ }^{31,33-36}$. Furthermore Mellaoui et al. ${ }^{37}$ have demonstrated that after a temperature increase, the previously non-volatile $\mathrm{SiO}_{\mathrm{x}} \mathrm{F}_{\mathrm{y}}$ becomes volatile and leads to smooth silicon sidewalls. Thus, cryogenic etching allows precise pattern transfer for sub $20 \mathrm{~nm}$ features with high aspect ratio. Variations of cRIE include e.g. a chopping-mode using $\mathrm{SF}_{6}$ and $\mathrm{O}_{2}$ for even higher aspect ratios and a generation of passivation layer by the injection of $\mathrm{SiF}_{4}$ and $\mathrm{O}_{2}$ to the gas chamber ${ }^{38,39}$.

Basically, reactive ion etching at cryogenic temperature suffers from the same effects, when it comes to high aspect ratio etching (aspect ratios >3:1). Figure 6 depicts the most significant of these aspect ratio dependent effects (ARDE), namely, RIE-lag, bowing (barreling), facetting and microtrenching. These effects are caused by several mechanisms, such as ion and kinetic neutral flux, electron shading, and transport and depletion of chemical etch and inhibit reactants. For further information and details about ARDE see ${ }^{31,40}$.
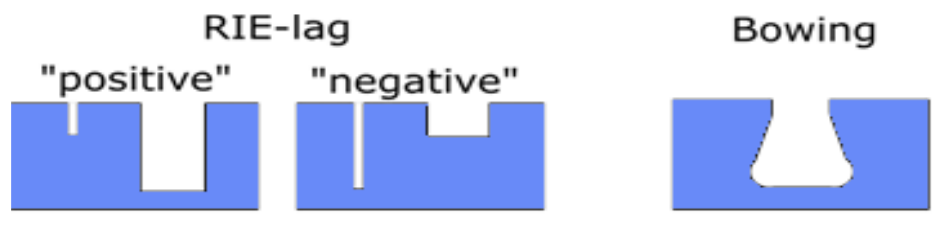
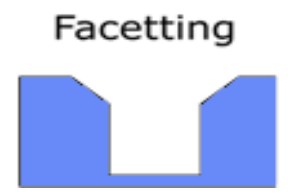

Microtrenching

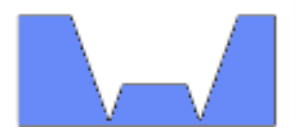

Figure 6: The most significant aspect ratio dependent effects. Figure prepared according to ${ }^{31}$. 
The etching recipe is as follows: $10 \mathrm{sccm} \mathrm{SF}_{6}, 2 \mathrm{sccm} \mathrm{O}_{2}$, process pressure $5 \mathrm{mTorr}$. A DC bias voltage of $70 \mathrm{~V}$ and an RF power of 11 Watt are kept constant. The wafer is fixed to the temperature-adjusted lower electrode with the substrate temperature being $-120^{\circ} \mathrm{C}$. The anisotropic etching behavior of this recipe was demonstrated in a previous publication ${ }^{1}$. Utilizing the recipe previously mentioned, a resist-to-silicon selectivity of $\sim 1: 4$ is obtained and combined with the anisotropic behavior, enables nano scale structures to be transferred into silicon by the use of $10 \mathrm{~nm}$ resist. Figure 7 shows examples of nanoelectronic devices fabricated by FE-SPL and cRIE.

a)
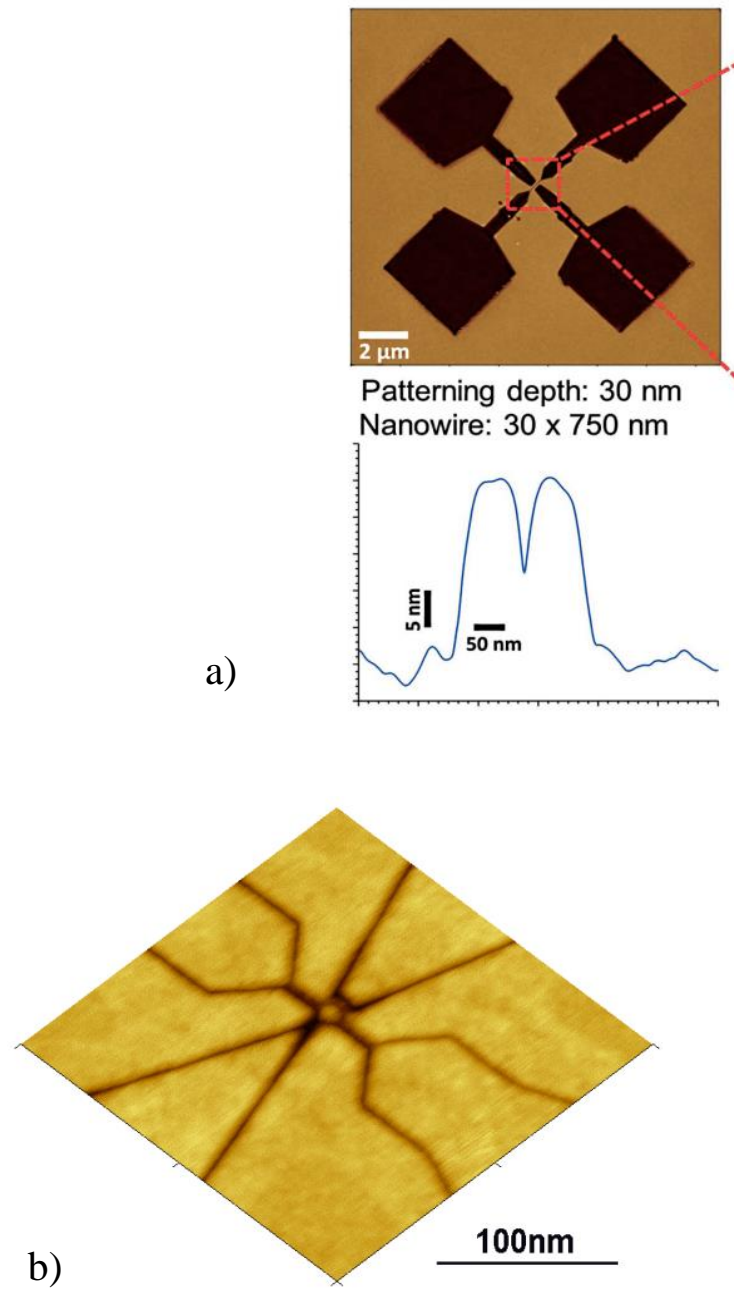
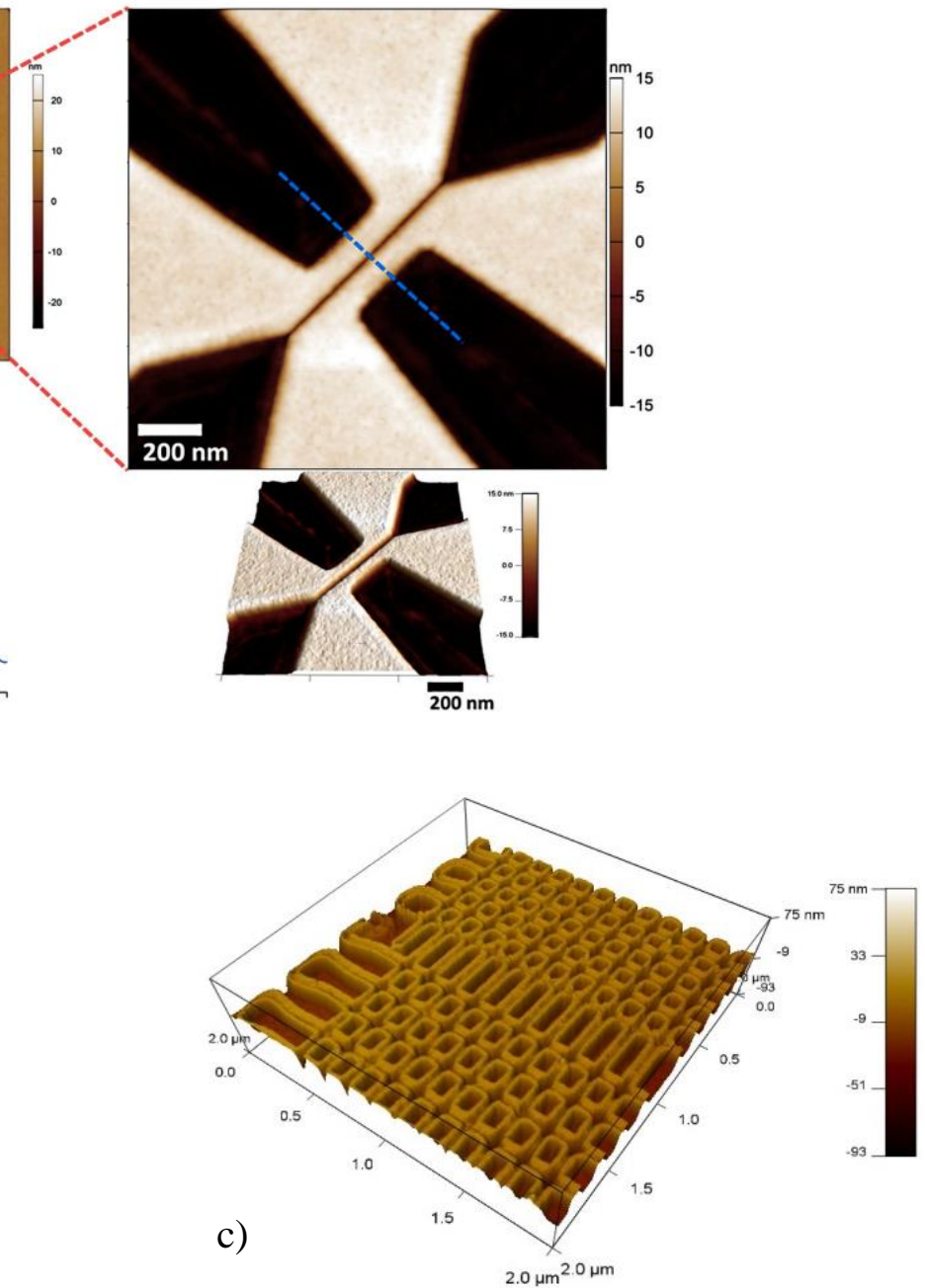

Figure 7: Examples of nanoelectronic devices fabricated by FE-SPL and cRIE. (a) NC-AFM images (directly after exposure) of a nanowire device patterned by multi-step FE-SPL in positive tone in 30nm thick calixarene resist. (b) Fast-AFM image of single electron transistors with nanosized Silicon-island as quantum dot obtained after etching. (d) NC-AFM image of plasmonic resonators ${ }^{14}$.

The dramatic improvements in speed, complexity and packing density, achieved through reduced device dimensions and new device structures, have enabled CMOS-based devices to meet the evolving requirements of integrated circuits for over 40 years. However, as minimum feature sizes have decreased and are targeted to fall $<10 \mathrm{~nm}$ and beyond ${ }^{41}$, crucial 
developments in lithographic tools have been essential. Important advances have been in high resolution lithographic and fabrication techniques, e.g. SPL ${ }^{42,43}$ as outlined above, $\mathrm{EBL}^{44,45}$, and $\mathrm{NIL}^{46}$, which have created alternative fabrication routes for 'beyond CMOS' $<10 \mathrm{~nm}$ electronic device structures. At the same time, challenging barriers are encountered in device physics, which have led to new structures ${ }^{47,48}$. However at scales $<10 \mathrm{~nm}$, quantum effects are increasingly likely to influence adversely the behaviour of all these devices. For example, the operation of a nominally 'classical' Fin-FET, with a fin only $\sim 4 \mathrm{~nm}$ wide, has been shown to be fundamentally limited by quantum confinement ${ }^{49}$. Although some researchers are exploring new materials such as or III-V and III-V on silicon ${ }^{50}$ graphene $^{51}, \mathrm{MoS}_{2}{ }^{52}$, there will be significant manufacturing problems with the inclusion of these technologies into any existing product line.

\section{FABRICATION OF SINGLE ELECTRON DEVICES}

Hence there are compelling reasons to establish new Si-based and CMOS compatible devices that can work at these dimensions. Fortunately at dimensions $<5 \mathrm{~nm}$, quantum effect devices such as single-electron (SE) devices in silicon ${ }^{53-55}$ look increasingly attractive. To observe these single electron effects, small silicon islands with diameters less than $10 \mathrm{~nm}$ have to be generated ${ }^{56}$ to produce quantum dots (QDs). These can be obtained by direct lithographic definition of the silicon island or by the point contact approach, whereby the point contacts are defined, and are then reduced in size by geometric controlled oxidation ${ }^{56,57}$.

Here, the second approach is followed. The SE devices were made on SOI wafer with a highly $\left(\sim 10^{20} / \mathrm{cm}^{3}\right) \mathrm{n}$-doped, ultrathin $(12 \pm 1 \mathrm{~nm})$ silicon layer on top of a $25 \mathrm{~nm}$ thick buried oxide layer. Contact pads and an area for device definition were defined by optical lithography and etching. Point contacts were defined as trenches into a 9-15 nm thick resist layer (AZ Barli or calixarene) by FE-SPL. The resist pattern was transferred into silicon using a $\mathrm{SF}_{6} / \mathrm{O}_{2}$ plasma with gas flows of 10 and $2.5 \mathrm{sccm}$ respectively, using cryo-etching with the sample cooled to $-120^{\circ} \mathrm{C}$ and a process pressure of 5 mTorr. AFM measurements were made prior to and post etching to control and optimize the process. After resist removal, a geometrically controlled oxidation step took place for quantum dot definition, as described in 56. Using photolithography and etching, patterning and point contact SET. Figure 8 (a) shows a SEM-image of the optical-lithography step for definition of contact pads. (b) Optical image, overlaid with AFM image, of the central region for FE-SPL. Four SETs have been defined in this area. (d) Sketch of point contact layout with opening angle $\alpha$ and neck width of d. Black lines indicate borders of the Si area and red lines marked FE-SPL defined lines. (d) High-resolution AFM image of the point-contact area defined by FE-SPL and prior to the oxidation step. The source/drain regions are on the left/right hand side of the device. [(a),(b),(d) from ${ }^{1}$ ].

Electrical characterization was performed using an Agilent 4155B parameter analyzer. Figures 9(a)-(d) show the corresponding drain-source conductance (gds) characteristics. The evidence for SET behavior of these devices at RT is seen in Fig. 9(a) indicated by the peak in the plot of gds vs. Vds. This corresponds to nonlinear asymmetric I-V characteristic at $290 \mathrm{~K}$. The conductance magnitude decreases as T is reduced to $200 \mathrm{~K}$ while the gds peak becomes more prominent [(b)-(c)]. For the reduced temperature of $150 \mathrm{~K}$ [Fig. 9(d)] the characteristics become more symmetrical. In this case, multiple conductance peaks [arrowed in (h)] are observed. By using the position of the first step, one can calculate the charging energy to be $\sim 0.17 \mathrm{eV}$ and a quantum dot diameter of $\sim 2 \mathrm{~nm}$.

\section{SUMMARY \& CONCLUSION}

In this paper we have demonstrated the promising combination of FE-SPL based high-resolution lithography with anisotropic resolution-preserving reactive ion etching at cryogenic temperature for the fabrication of nanoelectronic devices. Thereby, the active scanning probes used for FE-SPL are not only applied for lithography, but also for imaging and probing of the surface before and immediately after scanning probe patterning, enabling feature inspection, stitching and high accuracy overlay alignment. With these, atomic resolution at a low thermal noise floor is obtained routinely. The active cantilevers have enabled a compact system, suitable for the integration into a table-top scanning probe lithography tool to be realised. 

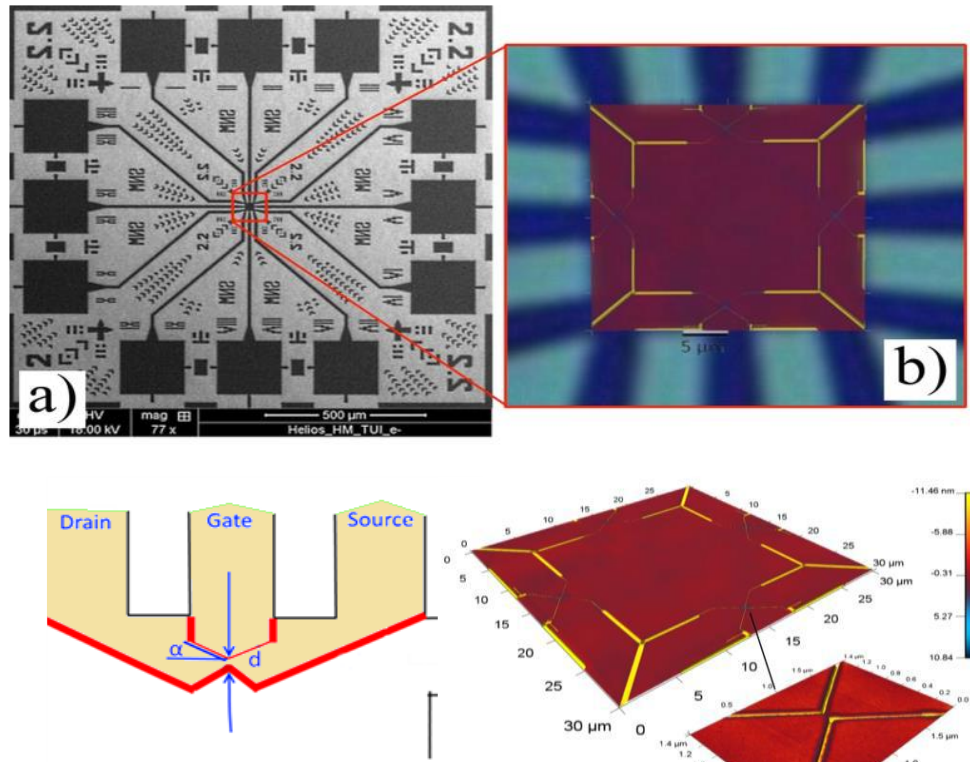

c)

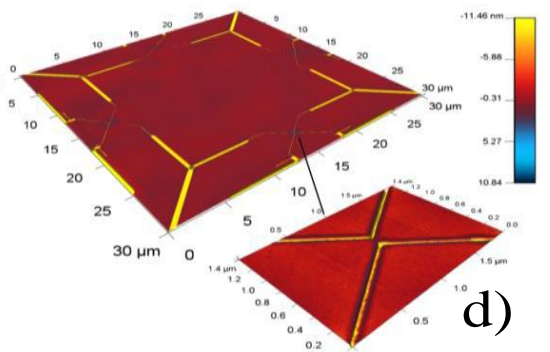

Figure 8: Principle of Mix and Match lithography

a)

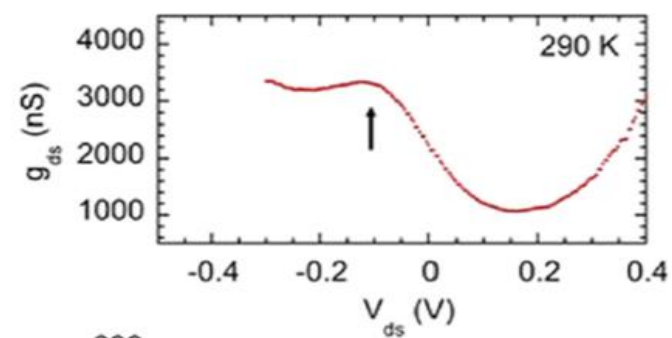

c)

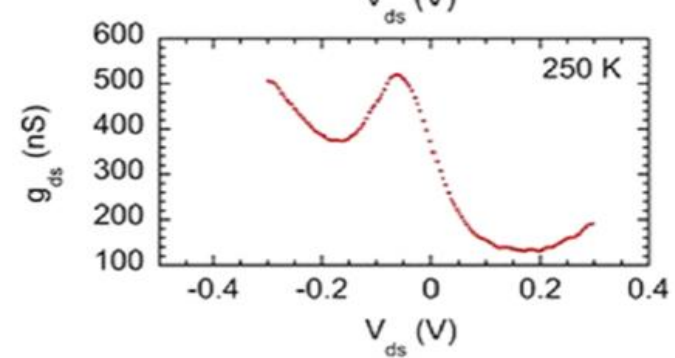

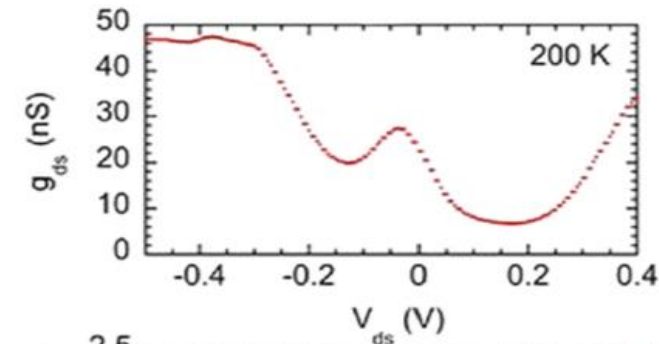

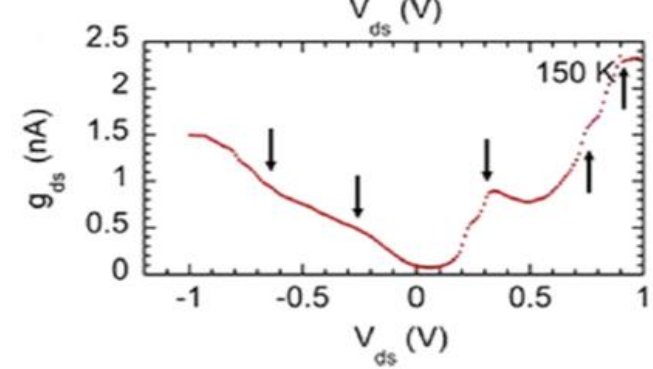

b)

d)

Figure 9: Electrical characteristics of a $30 \mathrm{~nm}$ wide point-contact SET, showing the $\mathrm{g}_{\mathrm{ds}}-\mathrm{V}_{\mathrm{ds}}$ characteristics, from 290 to 150 $\mathrm{K}$. Conductance oscillations, indicating the current steps, occur at the points indicated by arrows in the $150 \mathrm{~K}$ plot [from ${ }^{1}$ ].

To demonstrate the capabilities of this nanofabrication method for single electron transistors were created by FE-SPL, cRIE and controlled oxidation. Electrical characterization of these devices revealed single electron effects at room temperature. 
The combination of these techniques with high-throughput methods such as nanoimprint lithography could enable a fast and reproducible process chain for the routine fabrication of nanoelectronic, 'beyond CMOS' devices for the next generation electronics. Thereby, due to the merits of ambient condition operation, imaging and inspection capabilities, and throughput, FE-SPL could be the shortest way to get access to diverse single electron devices without employing capital equipment. Thus, the miniaturization of electronics to the single digit nanometer range and the possible use of SETs as a sensor of Q-bits in quantum computing becomes more possible.

\section{ACKNOWLEDGEMENT}

The research leading to these results has received funding from the European Union's Seventh Framework Programme FP7/2007-2013 under grant agreement no 318804 (Single Nanometer Manufacturing for beyond CMOS devices - acronym SNM). The authors thank Nikolay Nikolov for fruitful discussions and for the electronic design collaboration.

\section{REFERENCES}

[1] Rangelow, I. W., Ahmad, A., Ivanov, T., Kaestner, M., Krivoshapkina, Y., Angelov, T., Lenk, S., Lenk, C., Ishchuk, V., Hofmann, M., Nechepurenko, D., Atanasov, I., Volland, B., Guliyev, E., Durrani, Z., Jones, M., Wang, C., Liu, D., Reum, A., et al., "Pattern-generation and pattern-transfer for single-digit nano devices," JVST B 34(6), 06 K202 (2016).

[2] Müller, M., Fiedler, T., Gröger, R., Koch, T., Walheim, S., Obermair, C. and Schimmel, T., "Controlled structuring of mica surfaces with the tip of an atomic force microscope by mechanically induced local etching," Surf. Interface. Anal. 36, 189 (2004).

[3] Xu, S. and Liu, G. Y., "Nanometer-Scale Fabrication by Simultaneous Nanoshaving and Molecular Self-Assembly," Langmuir 13, 127 (1997).

[4] Custance, O., Perez, R. and Morita, S., “Atomic force microscopy as a tool for atom manipulation,” Nat. Nanotechnol. 4, 803 (2009).

[5] Mamin, H. J. and Rugar, D., "Thermomechanical writing with an atomic force microscope tip," Appl. Phys. Lett. 61, 1003 (1992).

[6] Hua, Y. M., King, W. P. and Henderson, C. L., "Nanopatterning Materials Using Area Selective Atomic Layer Deposition in Conjunction with Thermochemical Surface Modification via Heated AFM Cantilever Probe Lithography,” Microelectr. Eng. 85, 934 (1992).

[7] Martinez, R. V., Losilla, N. S., Martinez, J., Huttel, Y. and Garcia, R., "Patterning polymeric structures with $2 \mathrm{~nm}$ resolution at $3 \mathrm{~nm}$ half pitch in ambient conditions," Nano Lett. 7, 1846 (2007).

[8] Wilder, K., Quate, C. F., Adderton, D., Bernstein, R. and Elings, V., "Hybrid atomic force scanning tunneling lithography," Appl. Phys. Lett. 73, 2527 (1998).

[9] Stroscio, J. A. and Eigler, D. M., "Atomic and molecular manipulation with the scanning tunneling microscope," Science (80-. ). 254, 1319 (1991).

[10] Marrian, C. R. K., Dobisz, E. A. and Dagata, J. A., "Electron-Beam Lithography with the Scanning Tunneling Microscope," JVST B 10, 2877 (1992).

[11] Rolandi, M., Suez, I., Dai, H. and Frechet, J. M. J., "Dendrimer Monolayers as Negative and Positive Tone Resists for Scanning Probe Lithography," Nano Lett. 4(5), 889 (2004).

[12] Durrani, Z., Kaestner, M., Hofer, M., Ivanov, T. and Rangelow, I., "Scanning probe lithography for electronics at the 5nm scale," SPIE Newsroom, 2-4 (2013).

[13] Chou, S. Y., Krauss, P. R. and Renstrom, P. J., “Imprint Lithography with 25-Nanometer resolution,” Science (80-. ). 272, 85 (1996).

[14] Glinsner, T., Eibelhuber, M., Treiblmayr, D., Schamberger, B., Chouiki, M., Lenk, C., Krivoshapkina, Y., Hofmann, M., Lenk, S., Rangelow, I. W., DeMarneffe, J.-F., Chan, B. T., el Otell, Z. and Samara, V., "Wafer-Level 
Nanoimprint Lithography for Single Electron Transistors," Int. Conf. Nanoimprint Nanoprint Technol. (2017).

[15] Kaestner, M., Hofer, M. and Rangelow, I. W., "Nanolithography by scanning probes on calixarene molecular glass resist using mix-and-match lithography," J. Micro/Nanolithography, MEMS, MOEMS 12, 31111 (2013).

[16] Gotszalk, T., Grabiec, P. and Rangelow, I. W., "Piezoresistive sensors for scanning probe microscopy," Ultramicroscopy 82(1-4), 39 (2000).

[17] Pedrak, R., Ivanov, T., Gotszalk, T., Hudek, P., Fortagne, O. and Rangelow, I. W., "Micromachined AFM sensor with integrated piezoresistive sensor and thermal bimorph actuator for high-speed tapping-mode AFM and phaseimaging in higher eigenmodes," JVST B 21, 3102 (2003).

[18] Ahmad, A., Nikolov, N., Angelov, T., Ivanov, T., Reum, A., Atanasov, I., Guliyev, E., Ishchuk, V., Kaestner, M., Krivoshapkina, Y., Lenk, S., Lenk, C., Rangelow, I. W. and Holz, M., "Large area fast-AFM scanning with active 'Quattro' cantilever arrays," J. Vac. Sci. Technol. B, Nanotechnol. Microelectron. Mater. Process. Meas. Phenom. 34(6), 06KM03 (2016).

[19] Rangelow, I. W., Ivanov, T., Ahmad, A., Kaestner, M., Lenk, C., Bozchalooi, I. S., Xia, F., Youcef-Toumi, K., Holz, M. and Reum, A., "Active scanning probes: A versatile toolkit for fast imaging and emerging nanofabrication," JVST B 35, 06G101 (2017), doi.org/10.1116/1.4992073.

[20] Ishchuk, V., Guliyev, E., Aydogan, C., Buliev, I., Kaestner, M., Ivanov, T., Ahmad, A., Reum, A., Lenk, S., Lenk, C., Nikolov, N., Glinsner, T. and Rangelow, I. W., "Scanning probe-based high-accuracy overlay alignment concept for lithography applications," Appl. Phys. A Mater. Sci. Process. 123(1) (2017).

[21] Tachi, S., Tsujimoto, K. and Okudaira, S., "Low-temperature reactive ion etching and microwave plasma etching of silicon," Appl. Phys. Lett. 52, 616 (1998).

[22] Aydil, E. S., Gregus, J. A. and Gottscho, R. A., "Electron cyclotron resonance plasma reactor for cryogenic etching," Rev. Sci. Instrum. 64(12), 3572 (1993).

[23] Oehrlein, G. S. and Kurogi, Y., "Sidewall surface chemistry in directional etching processes,” Mat. Sci. R 24(4), 153 (1998).

[24] Aachboun, S. and Ranson, P., "Deep anisotropic etching of silicon," JVST A 17(4), 2270 (1999).

[25] Hofmann, M., Aydogan, C., Lenk, C., Krivoshapkina, Y., Lenk, S., Volland, B. E., Kaestner, M., Alaca, E. A., Manske, E. and Rangelow, I. W., "Selective Pattern Transfer of Nano-Scale Features Generated by FE-SPL in $10 \mathrm{~nm}$ Thick Resist Layers," Am. J. Nanoresearch Appl. (2018).

[26] Tachi, S., Tsujimoto, K., Arai, S. and Kure, T., "Low temperature dry etching," JVST A 9(3), 796 (1991).

[27] Winters, H. F. and Plumb, I. C., "Etching reactions for silicon with F atoms: Product distributions and ion enhancement mechanisms," JVST B 9, 197 (1991).

[28] Vasile, M. J. and Stevie, F. A., "Reaction of atomic fluorine with silicon: The gas phase products," J. Appl. Phys. 53, 3799 (1982).

[29] Shul, R. J., Hayes, T. R., Wetzel, R. C., Baiocchi, F. A. and Freund, R. S., "Electron impact ionization cross sections of SiF2," J. Chem. Phys. 89, 4042 (1988).

[30] Donelly, V. M., "Review Article: Reactions of fluorine atoms with silicon, revisited, again," JVST A A35, 05 C202 (2017).

[31] Rangelow, I. W., "Critical tasks in high aspect ratio silicon dry etching for micromechanical systems," JVST A 21(4), 1550 (2003).

[32] Mullins, C. B. and Coburn, J. W., "Ion beam assisted etching with fluorine at low temperatures," J. Appl. Phys. 76(11), 7562 (1994).

[33] Dussart, T., Tillocher, T., Lefaucheux, P. and Boufnichel, M., "Plasma cryogenic etching of silicon: from the early days to todays advanced technologies," J. Phys. D 47, 123001 (2014).

[34] Bartha, J. W., Greschner, J., Puech, M. and Maquin, P., "Low temperature etching of Si in high density plasma using SF6/O2," Microelectr. Eng. 27(1-4), 453 (1995).

[35] Rangelow, I. W., "Reactive ion etching for microelectrical mechanical system fabrication," JVST B 13(6), 2394 (1995).

[36] Olynick, D. L., Liddle, J. A., Harteneck, B. D., Cabrini, S. and Rangelow, I. W., "Nanoscale pattern transfer for templates, NEMS and nano-optics," Proc. SPIE 6462, 64620J1 (2007).

[37] Mellhaoui, X., Dussart, R., Tillocher, T., Lefaucheux, P., Ranson, P., Boufnichel, M. and Overzet, L. J., "SiOxFy 
passivation layer in silicon cryoetching,” J. Appl. Phys. 98(10), 104901 (2005).

[38] Tillocher, T., Dussart, R., Overzet, L. J., Mellhaoui, X., Lefaucheux, P., Boufnichel, M. and Ranson, P., "Two Cryogenic Processes Involving SF6, O2, and SiF4 for Silicon Deep Etching,” J. Electrochem. Soc. 155(3), D187 (2008).

[39] Isakovic, A. F., Evans-Lutterodt, K., Elliott, D., Stein, A. and Warren, J. B., “Cyclic, cryogenic, highly anisotropic plasma etching of silicon using SF6/O2," JVST A 26(5), 1182 (2008).

[40] Kassing, R. and Rangelow, I. W., "Etching processes for High Aspect Ratio Micro Systems Technology (HARMST)," Microsyst. Technol. 3(1), 20 (1996).

[41] H. Iwai, "Roadmap for 22nm and beyond", Microelectronic Eng., 86, no. 7-9, pp. 1520-1528, (2009)

[42] R. Garcia, A. W. Knoll, and E. Riedo, “Advanced scanning probe lithography”, Nature Nanotechnology, 9, 577 (2014).

[43] Z. Durrani, M. Jones, M. Kaestner, M. Hofer, E. Guliyev, A. Ahmad, T. Ivanov, J.-P. Zoellner, and I. W. Rangelow, "Scanning probe lithography approach for beyond CMOS devices", Proc. SPIE., 8680, p. 868017, (2013)

[44] B. E. Maile, W. Henschel, H. Kurz, B. Rienks, R Polman and P. Kaars, "Sub-10 nm Linewidth and Overlay Performance Achieved with a Fine-Tuned EBPG-5000 TFE Electron Beam Lithography System”, Jpn. J. Appl. Phys. 39, pp. 6836-6842 Part 1, No. 12B, (2000)

[45] V. R. Manfrinato, A. Stein, L. Zhang, C-Y. Nam, K. G. Yager, E. A. Stach and C. T. Black, "Aberration-Corrected Electron Beam Lithography at the One Nanometer Length Scale", ACS Nano Letters published (2017)

[46] M. C. Traub, W. Longsine, \& Van N. Truskett, "Advances in Nanoimprint Lithography", Annu. Rev. Chem. Biomol. Eng. 7, 583-604 (2016)

[47] D. Hisamoto, T Kaga, E. Takeda, "Impact of the Vertical SO1 "DELTA" Structure on Planar Device Technology" IEEE Trans. Electron Devices", 38 (6), pp1419-1424, (1991)

[48] V. S. Basker, T. Standaert, H. Kawasaki, C.-C. Yeh et al., "A $0.063 \mu \mathrm{m} 2$ FinFET SRAM cell demonstration with conventional lithography using a novel integration scheme with aggressively scaled fin and gate pitch", IEEE Symposium on VLSI Technology (VLSIT), Digest of Technical Papers, pp. 19-20, (2010)

[49] J. B. Chang, M. Guillorn, P.M. Solomon, C.-H. Lin, S.U. Engelmann, A. Pyzyna, J. A. Ott, W.E Haensch "Scaling of SOI FinFETs down to Fin Widths of $4 \mathrm{~nm}$ for the 10nm technology node", IEEE Symposium on VLSI Technology, Digest of Technical Papers, pp. 12-13, (2011)

[50] J. A. del Alamo, "Nanometre-scale electronics with III-V compound semiconductors", Nature, 479, no. 7373, pp. 317-323, (2011)

[51] K. Kim, J-Y Choi, T Kim, S-H Cho \& H-J Chung, "A role for graphene in silicon-based semiconductor devices", Nature, 479, no. 7373, pp. 338-344, (2011)

[52] Q. H. Wang, K. Kalanter-Zadeh, A. Kis, J. N. Coleman and M. S. Strano, "Electronics and optoelectronics of twodimensional transition metal dichalcogenides", Nature Nanotechnology, 7, pp. 699-712, (2012)

[53] Y. Takahashi, Y. Ono, A. Fujiwara, and H. Inokawa, "Silicon single-electron devices", J. Phys. Condens. Matter 14, pp. 995-1033, (2002).

[54] K. Likharev, "Single-electron devices and their applications", Proc. IEEE, 87, no. 4, pp. 606-632, (1999).

[55] Z. Durrani, "Single-Electron Devices and Circuits in Silicon", Imperial College Press, London, pp. $22-71$ (2009).

[56] Wang, C., Jones, M. E. \& Durrani, Z. A. K. "Single-electron and quantum confinement limits in length-scaled silicon nanowires", Nanotechnology 26, 305203 (2015)

[57] Durrani, Z. A. K., Jones, M. E., Wang, C., Liu, D. \& Griffiths, J. "Excited states and qua quantum confinement in room temperature few nanometre scale single electron transistors", Nanotechnology 28, 125208 (2017). 